\title{
INDIVIDUAL TREE DETECTION FROM UAV LIDAR DATA IN A MIXED SPECIES WOODLAND
}

\author{
A. Zaforemska ${ }^{1}$, W Xiao ${ }^{1}$, R. Gaulton ${ }^{1}$ \\ ${ }^{1}$ School of Engineering, Newcastle University, Newcastle-upon-Tyne, NE1 7RU, United Kingdom \\ (A.Zaforemska2, Wen.Xiao, Rachel.Gaulton)@ newcastle.ac.uk
}

KEY WORDS: UAV lidar, Single tree, Segmentation, Point cloud, Point intensity, Forest

\begin{abstract}
:
The study evaluates five existing segmentation algorithms to determine the method most suitable for individual tree detection across a species-diverse forest: raster-based region growing, local maxima centroidal Voronoi tessellation, point-cloud level region growing, marker controlled watershed and continuously adaptive mean shift. Each of the methods has been tested twice over one mixed and five single species plots: with their parameters set as constant and with the parameters calibrated for every plot. Overall, continuous adaptive mean shift performs best across all the plots with average F-score of 0.9 with fine-tuned parameters and 0.802 with parameters held at constant. Raster-based algorithms tend to achieve higher scores in coniferous plots, due to the clearly discernible tops, which significantly aid the detection of local maxima. Their performance is also highly dependent on the moving size window used to detect the local maxima, which ideally should be readjusted for every plot. Crown overlap, suppressed and leaning trees are the most likely sources of error for all the algorithms tested.
\end{abstract}

\section{INTRODUCTION}

Tree-level forest inventory has the potential to prove very useful in forest management, both in industry and natural conservation. Data on individual trees are important inputs for biomass estimation, biodiversity assessments and forest growth models. With the rapid development of remote sensing technologies and computing power, it is nowadays possible to extract and analyse forestry data on a sub-stand level and lidar is leading the way as the dominant technology for 3D mapping of forests. UAV lidar has attracted a lot of attention in the recent years as a less costly and more accessible alternative to airborne laser scanning (ALS), while producing very high density point clouds. The large volume of data produced by UAV lidar calls for improvement of methodologies of point cloud processing and feature extraction. Individual tree features extracted from lidar data can be a useful input for species classification (Dalponte et al., 2016; Fassnacht et al., 2016). In order to achieve this, segmentation needs to be performed using method achieving high precision across a wide variety of species.

A number of algorithms have been developed over the years for segmentation of lidar point clouds collected over forests to delineate individual trees. Some of the most popular ones employ region growing (Dalponte and Coomes, 2016; Solberg et al., 2006) watershed delineation (Chen et al. 2006) and clustering (Ferraz et al., 2012; Gupta et al., 2010; Lindberg et al., 2014; Xiao et al., 2016). However, despite the abundance of available algorithms, the vast majority produce reliable results only under very specific conditions e.g. specific species. Different processing methods have varied performance in different types of forests, as they make assumptions about the spacing between the trees, their shape and size. Most of them have been developed specifically for conifers (Hamraz et al., 2016). Coniferous trees often have one characteristic top of the crown, which makes it easier to detect using local maxima based methods. Variability of height within the tree crowns, characteristic for many broadleaf species, is one of the main sources of error for region growing and watershed algorithms (Hamraz et al., 2016).

A number of studies (Kaartinen et al., 2012; Vauhkonen et al., 2012; Eysn et al., 2015) have reviewed several segmentation algorithms in a variety of environments, but have not explored the effect of species-dependant individual tree structural parameters or heterogeneity of the data such as point cloud density, on the algorithm performance.

The study is focused on tree segmentation from a point cloud obtained by a UAV lidar system flown over mixed temperate forest, in order to determine the method most suitable for single tree detection across variety of species. The final goal is to create a dataset of individual trees on which delineation and species classification can be performed.

\section{METHODOLOGY}

\subsection{Study area}

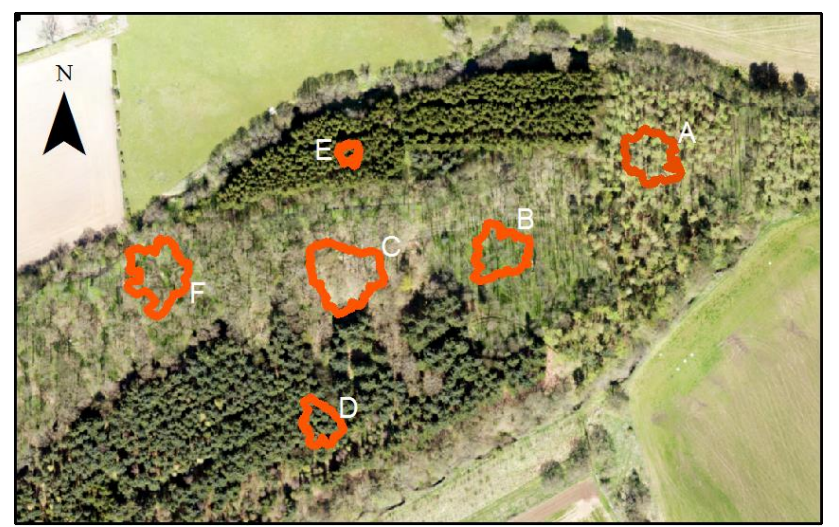

Figure 1. Aerial view of Hangingleaves Wood in Cockle Park Farm, Northumberland, with six plots: A - European larch, B Sycamore, C-English oak, D - Sitka spruce, E - Norway spruce, F- mixed. 
The site chosen for the study is Hangingleaves Wood in Cockle Park Farm near Morpeth, Northumberland. The farm is property of Newcastle University, which enables an easy access to the study area. Hangingleaves Wood is a woodland consisting of 5 distinct tree species: European larch (Larix decidua), Sitka spruce (Picea sitchensis), Sycamore (Acer pseudoplatanus), Norway spruce (Picea abies) and English oak (Quercus robur). Most of the woodland is divided into single species areas, which allows for an easy establishment of the plots, as seen in Figure 1. Six plots have been defined in the site, five single-species and one mixed (Sycamore and English oak). Ground truth data such as tree position, height and crown diameter, has been provided by a previous study in the same area (Berra et al., 2019).

\subsection{Data collection}

UAV lidar data has been collected using ROBINI MINI UAV lidar system in December 2018. The sensor used was RIEGL miniVUX-1UAV, a lightweight laser scanner developed specifically for UAVs, with accuracy of $15 \mathrm{~mm}$ and precision of $10 \mathrm{~mm}$. ROBIN MINI can achieve very high point cloud density, up to 90 points $/ \mathrm{m}^{2}$, depending on elevation and speed of the flight. For purpose of this study, the UAV was flown at $60 \mathrm{~m}$ height, at constant speed of $8 \mathrm{~m} / \mathrm{s}$, with stabilized seesaw trajectory. The resulting point cloud has density of approximately 50 points $/ \mathrm{m}^{2}$.

\subsection{Data pre-processing}
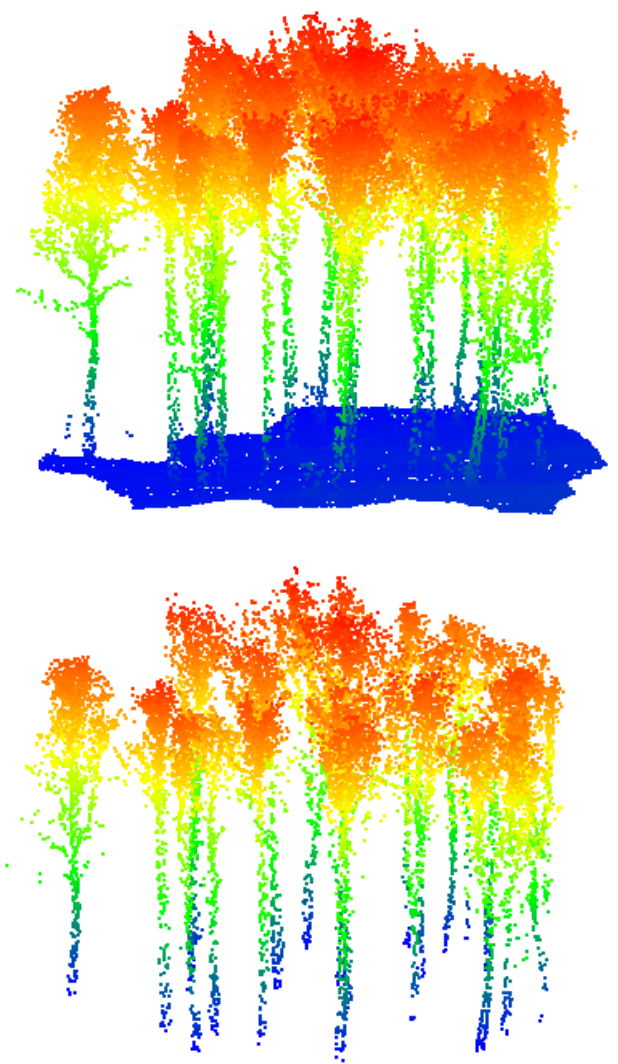

Figure 2. Plot B (Sycamore), top - before pre-processing, bottom - after pre-processing.

The lidar data has been quality checked for coverage and spatial accuracy and obtained scores within the range recommended by processing guidelines. Further calibration was conducted using
TerraSolid software package. Classification of the ground points allowed for height normalization. To improve the quality, data has been denoised and filtered for high intensity points. The high intensity points are used only to emphasize certain aspects of the tree structure such as trunks that could facilitate the segmentation. This paper does not focus on exact intensity values and reflectivity of the targets, hence no radiometric calibration was performed. Points up to 2 meters from the ground level were not included in tree detection, as they belong to bushes and grass that are out of interest. The effects of pre-processing on the point cloud are presented in Figure 2.

\subsection{Segmentation methods}

Five different existing algorithms have been used to segment the point cloud and detect the trees. Each of the methods has been originally developed using data from a specific type of forest. Three of the methods require smoothed canopy height models (CHMs) in order to identify the trees, while two methods work directly on the point cloud. All the raster-based methods require the input of CHM spatial resolution and the size of moving window to identify the local maxima. These parameters were set at default of $0.5 \mathrm{~m}$ and $4 \mathrm{~m}$ respectively. Methods 1-4 are available through lidR R package (Roussel et al., 2019), while Method 5 has been provided by one of the authors. Adjustable parameters have been listed under each method, with default values given in parenthesis.

Method 1 (Dalponte and Coomes, 2016): raster-based local maxima region growing, developed using data from Alpine mixed forest and methods used by Hyyppa et al., 2001. finds local maxima within a CHM to work as initial points to which the neighbouring crown points are added through a decision tree, based on two thresholds: if the vertical distance between the point and the maximum is less than a defined percentage of total tree height (threshold 1) and if the point's height is greater than the average height of the region multiplied by a set number (threshold 2). The process continues until all the points have been segmented. Parameters: growing threshold 1 (0.45), growing threshold 2 (0.55), maximum crown diameter (10 pixels), minimum height of the tree $(2 \mathrm{~m})$.

Method 2 (Silva et al., 2016): CHM based local maxima Voronoi tessellation, developed for segmentation and delineation of longleaf pine (Pinus palustris). The algorithm applies a buffer around the local maxima, calculating its diameter by multiplying the tree height by 0.6 (the value can be adjusted by the user), in order to delineate the tree crowns. The points are further split into separate tree classes through Voronoi tessellation, with local maxima as centroids. Parameters: maximum crown diameter as proportion of the tree height $(0.6)$.

Method 3 (Li et al., 2012): Point cloud level region growing, developed for use in mixed coniferous forests, uses horizontal spacing between the points and separate trees. Moving from the top of the tree downwards, the horizontal distance between the unclassified point and the nearest point already assigned to a tree is compared with a set threshold. Points that are further apart than the threshold are excluded from a tree. There are two different thresholds, used depending on the height of the point, to account for the shape of crown. Dt1 is used if point height is lower than the set elevation parameter $Z u, D t 2$ - if the point height is higher. Parameters: dt1 (1.5), dt2 (2), search radius (2 m), Zu (15 m), minimum height of the tree $(2 \mathrm{~m})$. 
Method 4 (Chen et al., 2006): Marker controlled watershed algorithm, developed in open oak savanna woodland. Assuming the tree tops to be in the centre of the crowns and uses them as markers which indicate separate 'catchment zones. Tree crowns can be delineated using a flooding simulation on the reverse CHM (with the local maxima serving as minima). The areas which are first to flood point to separate trees. Parameters: minimum height of the tree $(2 \mathrm{~m})$.

Method 5 (Xiao et al., 2016): Continuously Adaptive Mean Shift (CamShift), based on the mean shift clustering algorithm applied to urban trees. Applied directly to the point cloud, seeks areas of highest point density - in case of trees it is usually the top of the crown. Size of the moving window is determined by a parameter called bandwidth. Assuming that the width of the crown depends on the height of the tree, bandwidth is adaptable to the absolute height of the kernel centre. The 3D kernel is based on the Pollock tree model (Pollock, 1994), with shape determined by $n$ (value between 1 and 2, where 1 is a cone and 2 is an ellipsoid). Parameters: bandwidth (5), $\mathrm{n}(1.5)$.

Two tests have been conducted to assess the methods. First test used parameters calibrated for best performance in each individual plot, in order to further examine the full capabilities of each method. Second test sets all the parameters of each algorithm as constant across all the plots. It is necessary, as broad applicability to a range of tree species without prior knowledge is required if the methods are to be used prior to species classification of the whole woodland.

All the segmented point clouds were visually assessed and validated against the ground measurements.

\section{RESULTS AND DISCUSSION}

The outcomes of segmentation with calibrated parameters are presented in Table 1, with constant parameters - Table 2. Figures 1-6 represent front and the top view of every plot as a point cloud, showing examples of segmentation by each method tested.

\subsection{Plot A: European larch (Larix decidua)}

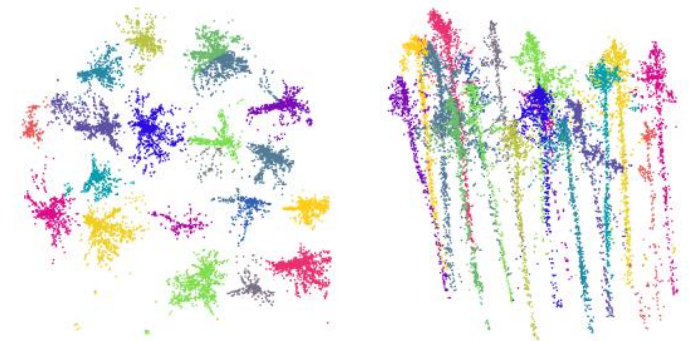

Figure 1. Top and the front of Plot A segmented using Method 2 (calibrated), with each separate tree assigned a random colour.

European larch (Figure 1) is unique among the trees included in the study as the only deciduous conifer. The plot achieved highest average detection accuracy in the calibrated test and the second highest for non-calibrated test (mean F-score of all the methods approx. 0.9 and 0.85 respectively), which indicates it is one of the easiest to segment. That is due to both its shape, with a clearly discernible apex often found in conifers, as well as its deciduous nature which in the leaf-off season allows for detection of trunks. Test 2 achieved similar results across all the methods, with Methods 1 and 2 achieving the highest F-score of 0.865 . Method 5 achieved the lowest score due to high oversegmentation, most likely owing to the detection of elements of understorey.

\begin{tabular}{|c|c|c|c|c|c|c|}
\hline \multicolumn{7}{|c|}{ A. Larch } \\
\hline & $\mathrm{TP}$ & FP & FN & $\mathrm{P}$ & $\mathrm{R}$ & F-score \\
\hline Dalponte2016 & 19 & 4 & 1 & 0.826 & 0.950 & 0.884 \\
\hline Silva2016 & 19 & 3 & 1 & 0.864 & 0.950 & 0.905 \\
\hline Li2010 & 18 & 2 & 2 & 0.900 & 0.900 & 0.900 \\
\hline Chen2006 & 16 & 1 & 4 & 0.941 & 0.800 & 0.865 \\
\hline Xiao2016 & 19 & 0 & 1 & 1.000 & 0.950 & 0.974 \\
\hline \multicolumn{7}{|c|}{ B. Sycamore } \\
\hline & $\mathrm{TP}$ & FP & $\mathrm{FN}$ & $\mathrm{P}$ & $\mathrm{R}$ & F-score \\
\hline Dalponte2016 & 19 & 3 & 1 & 0.864 & 0.950 & 0.905 \\
\hline Silva2016 & 18 & 3 & 2 & 0.857 & 0.900 & 0.878 \\
\hline Li2012 & 19 & 5 & 1 & 0.792 & 0.950 & 0.864 \\
\hline Chen2006 & 18 & 2 & 2 & 0.900 & 0.900 & 0.900 \\
\hline Xiao2016 & 20 & 1 & 0 & 0.952 & 1.000 & 0.976 \\
\hline \multicolumn{7}{|c|}{ C. Oak } \\
\hline & $\mathrm{TP}$ & FP & FN & $\mathrm{P}$ & $\mathrm{R}$ & F-score \\
\hline Dalponte2016 & 15 & 2 & 5 & 0.882 & 0.750 & 0.811 \\
\hline Silva2016 & 16 & 4 & 4 & 0.800 & 0.800 & 0.800 \\
\hline Li2012 & 14 & 2 & 6 & 0.875 & 0.700 & 0.778 \\
\hline Chen2006 & 15 & 3 & 5 & 0.833 & 0.750 & 0.789 \\
\hline Xiao2016 & 17 & 2 & 3 & 0.895 & 0.850 & 0.872 \\
\hline \multicolumn{7}{|c|}{ D. Sitka spruce } \\
\hline & $\mathrm{TP}$ & FP & FN & $\mathrm{P}$ & $\mathrm{R}$ & F-score \\
\hline Dalponte2016 & 17 & 1 & 3 & 0.944 & 0.850 & 0.895 \\
\hline Silva2016 & 17 & 2 & 3 & 0.895 & 0.850 & 0.872 \\
\hline Li2012 & 17 & 5 & 3 & 0.773 & 0.850 & 0.810 \\
\hline Chen2006 & 19 & 2 & 1 & 0.905 & 0.950 & 0.927 \\
\hline Xiao2016 & 18 & 1 & 2 & 0.947 & 0.900 & 0.923 \\
\hline \multicolumn{7}{|c|}{ E. Norway spruce } \\
\hline & TP & FP & $\mathrm{FN}$ & $\mathrm{P}$ & $\mathrm{R}$ & F-score \\
\hline Dalponte2016 & 19 & 2 & 1 & 0.905 & 0.950 & 0.927 \\
\hline Silva2016 & 17 & 3 & 3 & 0.850 & 0.850 & 0.850 \\
\hline Li2010 & 19 & 2 & 1 & 0.905 & 0.950 & 0.927 \\
\hline Chen2006 & 18 & 2 & 2 & 0.900 & 0.900 & 0.900 \\
\hline Xiao2016 & 17 & 2 & 3 & 0.895 & 0.850 & 0.872 \\
\hline \multicolumn{7}{|c|}{ F. Mix } \\
\hline & $\mathrm{TP}$ & FP & FN & $\mathrm{P}$ & $\mathrm{R}$ & F-score \\
\hline Dalponte2016 & 18 & 3 & 2 & 0.857 & 0.900 & 0.878 \\
\hline Silva2016 & 19 & 4 & 1 & 0.826 & 0.950 & 0.884 \\
\hline Li2010 & 18 & 3 & 2 & 0.857 & 0.900 & 0.878 \\
\hline Chen2006 & 16 & 4 & 4 & 0.800 & 0.800 & 0.800 \\
\hline Xiao2016 & 17 & 1 & 3 & 0.944 & 0.850 & 0.895 \\
\hline
\end{tabular}

Table 1. Results of tree detection across all the plots with parameters calibrated for the best outcome (Test 1). TP - true positive, $\mathrm{FP}$ - false positive, $\mathrm{FN}$ - false negative, $\mathrm{P}$ - precision, $\mathrm{R}$ - recall 


\begin{tabular}{|l|r|r|r|c|c|r|}
\hline \multicolumn{7}{|c|}{ A. Larch } \\
\hline & TP & FP & FN & P & R & F-score \\
\hline Dalponte2016 & 16 & 1 & 4 & 0.941 & 0.800 & 0.865 \\
\hline Silva2016 & 16 & 1 & 4 & 0.941 & 0.800 & 0.865 \\
\hline Li2010 & 18 & 5 & 2 & 0.783 & 0.900 & 0.837 \\
\hline Chen2006 & 16 & 2 & 4 & 0.889 & 0.800 & 0.842 \\
\hline Xiao2016 & 18 & 6 & 2 & 0.750 & 0.900 & 0.818 \\
\hline
\end{tabular}

B. Sycamore

\begin{tabular}{|l|r|r|r|c|c|r|}
\hline & TP & FP & FN & P & R & F-score \\
\hline Dalponte2016 & 15 & 0 & 5 & 1.000 & 0.750 & 0.857 \\
\hline Silva2016 & 15 & 0 & 5 & 1.000 & 0.750 & 0.857 \\
\hline Li2010 & 19 & 7 & 1 & 0.731 & 0.950 & 0.826 \\
\hline Chen2006 & 15 & 0 & 5 & 1.000 & 0.750 & 0.857 \\
\hline Xiao2016 & 18 & 0 & 2 & 1.000 & 0.900 & 0.947 \\
\hline
\end{tabular}

\section{Oak}

\begin{tabular}{|l|r|r|r|c|c|r|}
\hline & TP & FP & FN & P & R & F-score \\
\hline Dalponte2016 & 16 & 7 & 4 & 0.696 & 0.800 & 0.744 \\
\hline Silva2016 & 17 & 8 & 3 & 0.680 & 0.850 & 0.756 \\
\hline Li2010 & 16 & 15 & 4 & 0.516 & 0.800 & 0.627 \\
\hline Chen2006 & 16 & 6 & 4 & 0.727 & 0.800 & 0.762 \\
\hline Xiao2016 & 15 & 5 & 2 & 0.750 & 0.882 & 0.811 \\
\hline
\end{tabular}

\section{Sitka spruce}

\begin{tabular}{|l|r|r|r|c|c|r|}
\hline & TP & FP & \multicolumn{1}{|c|}{ FN } & P & R & F-score \\
\hline Dalponte2016 & 14 & 0 & 6 & 1.000 & 0.700 & 0.824 \\
\hline Silva2016 & 14 & 0 & 6 & 1.000 & 0.700 & 0.824 \\
\hline Li2010 & 15 & 3 & 5 & 0.833 & 0.750 & 0.789 \\
\hline Chen2006 & 16 & 0 & 6 & 1.000 & 0.727 & 0.842 \\
\hline Xiao2016 & 12 & 0 & 8 & 1.000 & 0.600 & 0.750 \\
\hline
\end{tabular}

\section{E. Norway spruce}

\begin{tabular}{|l|r|r|r|c|c|r|}
\hline & TP & FP & FN & P & R & F-score \\
\hline Dalponte2016 & 7 & 0 & 13 & 1.000 & 0.350 & 0.519 \\
\hline Silva2016 & 7 & 0 & 13 & 1.000 & 0.350 & 0.519 \\
\hline Li2010 & 9 & 1 & 11 & 0.900 & 0.450 & 0.600 \\
\hline Chen2006 & 7 & 0 & 13 & 1.000 & 0.350 & 0.519 \\
\hline Xiao2016 & 9 & 0 & 11 & 1.000 & 0.450 & 0.621 \\
\hline
\end{tabular}

\section{F. Mix}

\begin{tabular}{|l|r|r|r|c|c|r|}
\hline & TP & FP & FN & P & R & F-score \\
\hline Dalponte2016 & 16 & 4 & 2 & 0.800 & 0.889 & 0.842 \\
\hline Silva2016 & 17 & 7 & 3 & 0.708 & 0.850 & 0.773 \\
\hline Li2010 & 18 & 15 & 2 & 0.545 & 0.900 & 0.679 \\
\hline Chen2006 & 17 & 7 & 3 & 0.708 & 0.850 & 0.773 \\
\hline Xiao2016 & 16 & 4 & 1 & 0.800 & 0.941 & 0.865 \\
\hline
\end{tabular}

Table 2. Results of tree detection across all the plots with parameters set as constant (Test 2). TP indicates true positive correctly detected, FP - false positive, oversegmentation, FN false negative, undersegmentation, $\mathrm{P}$ - precision, $\mathrm{R}$ - recall.
After calibration, Method 5 performed the best, with F-score of 0.974 and no false positives. One case of undersegmentation occurred in all the processed plots, due to one of the trees leaning on another, which causes the two tree crowns to appear as one.

\subsection{Plot B: Sycamore (Acer pseudoplatanus)}
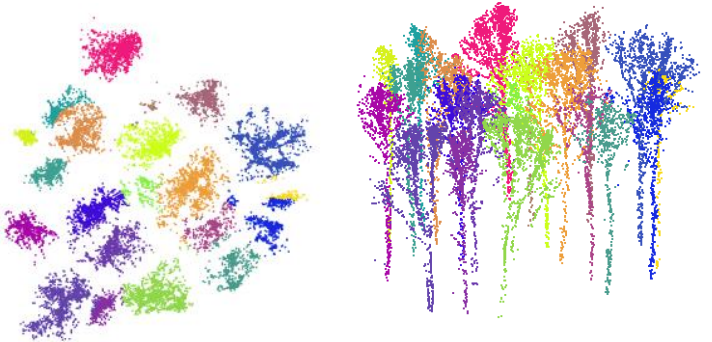

Figure 2. Top and the front of Plot B segmented using Method 5 (calibrated), with each separate tree assigned a random colour.

Sycamores (Figure 2) in plot B have relatively narrow crowns with very little overlap between the adjacent trees, which explains relatively low level of undersegmentation in comparison with the other plots in Test 1 . Instances of oversegmentation are more likely due to the branching structure, where two major branches of one tree are identified as two different specimens. It is the plot with highest level of detection accuracy in Test 2, but lower recall than in the case of Test 1 , as the CHM resolution is too low and size of the kernel too large for the plot. Method 5 obtained the highest accuracy scores in both tests (F-score > 0.9).

\subsection{Plot C: English oak (Quercus robur)}

Plot C is characterized by broad, spread out crowns (Figure 3). This feature might explain the larger amount of overdetected trees in Test 2 in comparison with the other plots (up to 15 in case of Method 3). It is more difficult to establish local maxima in trees with broad crowns as they do not have one characteristic

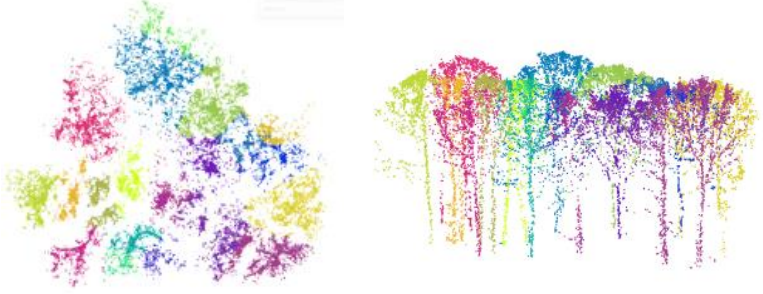

Figure 3. Top and the front of Plot C segmented using Method 3 (calibrated), with each separate tree assigned a random colour.

peak. Parameter calibration help to significantly reduce the number of overdetected trees, but did not improve the recall. Underdetection in plot $\mathrm{C}$ is mot likely due to some overlap between the crowns. Method 5 achieved the best accuracy in both tests, but its performance after calibration did not increase significantly.

\subsection{Plot D: Sitka spruce (Picea sitchensis)}

Due to the presence of foliage, only very small number of trunk points could be retrieved, not sufficient to significantly aid tree detection (Figure 4). In both tests, Method 4 achieves the best result $(\mathrm{F}$-score $=0.842$ and $\mathrm{F}$-score $=0.927)$. Method 5 notes the poorest performance from all the algorithms in Test 2 , but improves significantly after parameter calibration (from Fscore $=0.750$ to $\mathrm{F}$-score $=0.923$ ). 


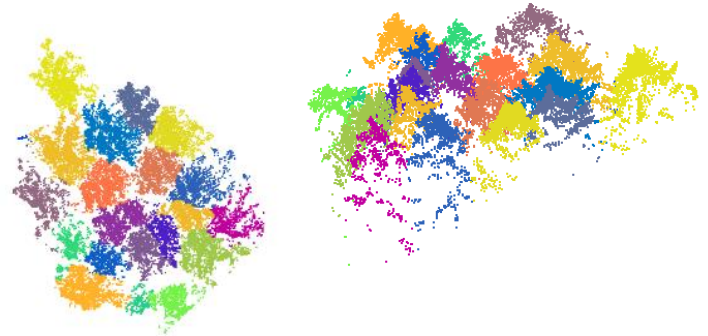

Figure 4. Top and the front of Plot D segmented using Method 4 (calibrated), with each separate tree assigned a random colour.

In Test 2 the majority of errors were false negative and only Method 3 resulted in false positive (3 overdetections). After calibration, the number of underdetections was reduced, while overdetections increased as some isolated points in the lower sections of the crowns were classified as separate trees.

\subsection{Plot E: Norway spruce (Picea abies)}

There are no obvious structural differences between Norway and Sitka spruce that are visible in the point cloud, however, the plot is smaller in area and as a result, more dense. As in case of Sitka spruce, the foliage prevents from detection of trunks and lower parts of the crown (Figure 5). Those two factors combined make it more difficult to detect single trees without fine-tuning the parameters. In Test 2 the methods obtained relatively poor results, with average F-score of 0.555 . The highest F-score of 0.621 was achieved by Method 1 . All the algorithms significantly undersegmented the plot, with up to 13 undetected trees for methods 1,2 and 4 . The accuracy improved by a large margin after the calibration with average F-score of 0.895 in Test 1. Method 1 and 3 obtained the best result (F-score $=0.927$ ), likely due to the conical shape of the crown, which aids the detection of local maxima.
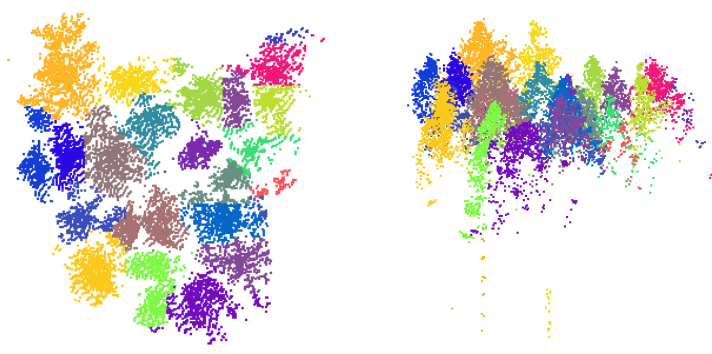

Figure 5. Top and the front of Plot E segmented using Method 1 (calibrated), with each separate tree assigned a random colour.

\subsection{Plot F: Mix (Sycamore and English oak)}

The plot combines the properties of plots B and C, with most of the errors occurring due to crown overlap and spread out branches (Figure 6). The average segmentation accuracy is higher than for plot $\mathrm{C}$, but lower than for plot B with Fscore $=0.867$ for Test 1 and F-score $=0.786$ for Test 2 . Parameter calibration slightly improves the performance of most of the algorithms, apart from Method 3 for which the accuracy improved significantly (from F-score $=0.679$ to F-score $=0.878$ ). In both of the tests, Method 5 achieved the best results. One underdetection error was present across all the methods due to a suppressed tree with limited number of points in the centre of the plot.
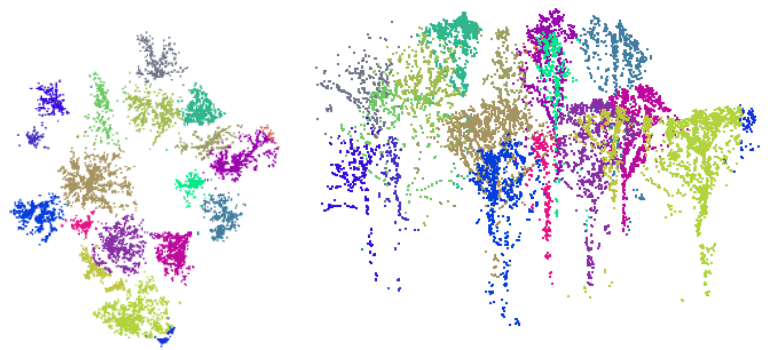

Figure 6. Top and the front of Plot A segmented using Method 5 (calibrated), with each separate tree assigned a random colour.

\subsection{Overall performance of the algorithms}

Figure 7 shows the average performance results for each of the algorithms, with and without calibrated parameters. In both cases Method 5 achieves the best overall accuracy (F-score $=0.919$ for calibrated and F-score $=0.802$ for uncalibrated), followed by Method $1(\mathrm{~F}$-score $=0.883$ for calibrated and F-score $=0.775$ for uncalibrated). Method 5 obtained the highest scores in four plots in Test 1 and three plots in Test 2 out of six plots in total, making it the best performing algorithm across a variety of tree species. Method 5 is unique in the group, as the only clustering algorithm and one of two working directly on the point cloud. As it operates in 3D it has a significant advantage over raster-based methods. Clustering algorithms are proven to work effectively across a variety of vegetation types: urban, temperate and tropical forests (Xiao et al., 2016; Ferraz et al., 2012, Ferraz et al., 2016).
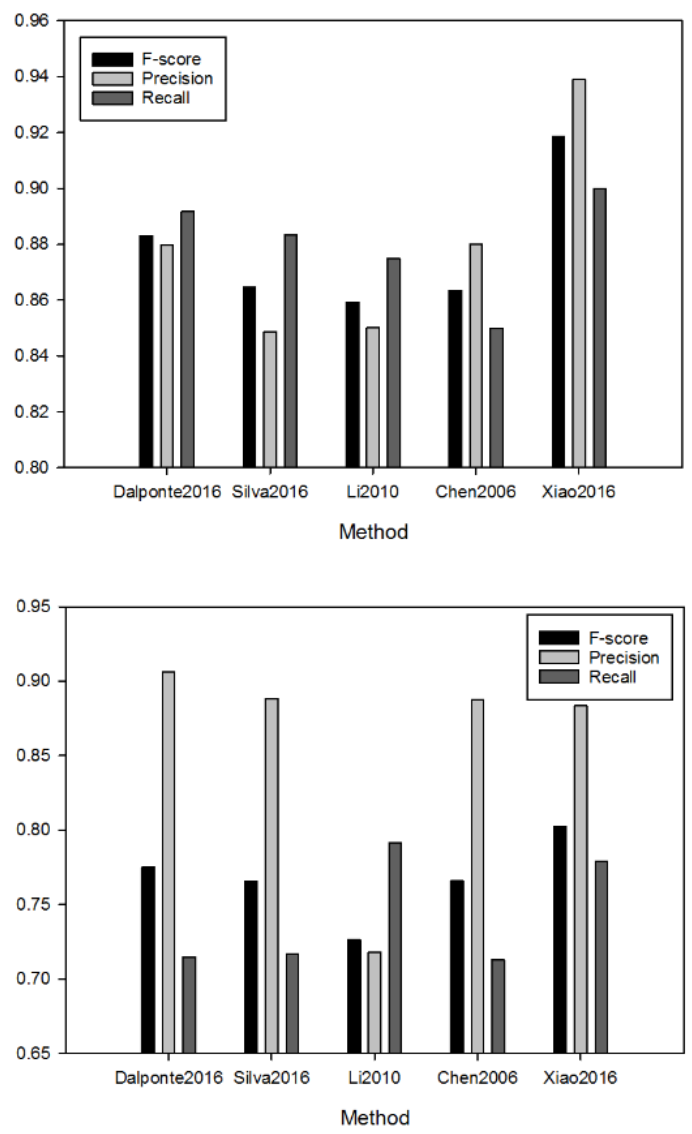

Figure 7. Average F-score, precision and recall for each algorithm averaged over all of the plots. Top: fine-tuned parameters, bottom: constant parameters. 
However, certain degree of calibration is still necessary to obtain the best results - size and shape of the 3D kernel play a crucial role in accurate tree detection and delineation. Even with careful fine-tuning of the kernel parameters, the algorithm may not always detect separate trees if they are leaning or their crowns are overlapping. This is also the case in other methods (Figure 8).

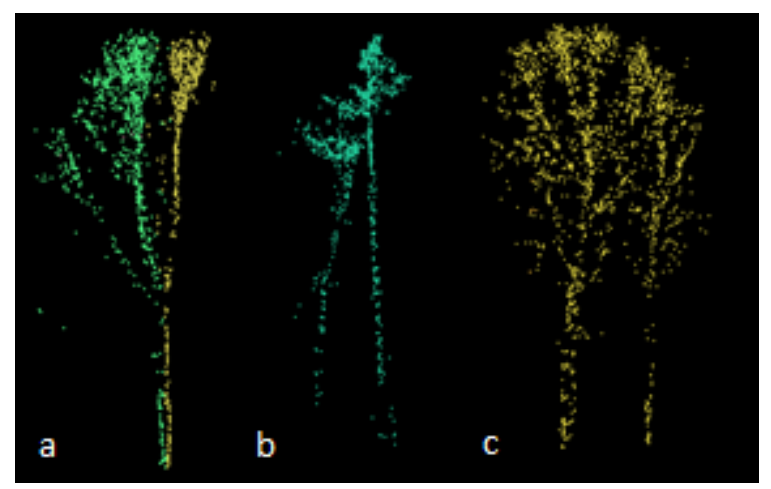

Figure 8. Examples of common segmentation errors: a oversegmentation due to diverging branches (plot B), $\mathrm{b}-$ undersegmentation due to leaning tree (plot A), c undersegmentation due to crown overlap (plot C).

The three raster-based approaches tested in this study achieved similar level of accuracy, their performance highly dependent on the tree species and parameter calibration. Canopy height models (CHM) represent three dimensional reality in 2D causing significant oversimplification and loss of information (Ferraz et al., 2012, Kandare et al., 2016). Still, CHM based algorithms are some of the most popular segmentation methods, as they allow for easy identification of local maxima (Figure 2). This explains the high scores of raster-based algorithms among the species with conical shape and clearly defined tree tops, particularly Sitka spruce and Norway spruce.

However, those methods may struggle with broadleaf trees which have wide spread out crowns, usually without one characteristic top point. This may result in oversegmentation, when several major branches within one specimen, with high degree of separation at the top of the crown are classified as separate trees. The problem also applies to point cloud-based region growing algorithm (Method 3). Size of the kernel used to detect local maxima also has a significant impact on the accuracy of tree detection and the raster-based methods achieved higher scores when the kernel size was adapted to the particular plot (Figure 9).

Only one suppressed tree has been identified in this study (plot F) and it was not detected by any of the methods tested. The number of points representing the tree was too low to allow for detection. Furthermore, CHMs often omit suppressed and intermediate trees, as they represent the uppermost layer of the canopy (Kandare et al., 2016). Previous studies have established it is possible for clustering-based approaches to successfully detect subdominant trees with high level of accuracy, but a sufficient number of points is necessary (Eysn et al. 2015).

The exposure of trunks and branching structure in the deciduous specimens aids the visual assessment and validation of the segmentation results, however further tests on leaf-on data are necessary to determine whether it has a significant impact on the segmentation process itself.
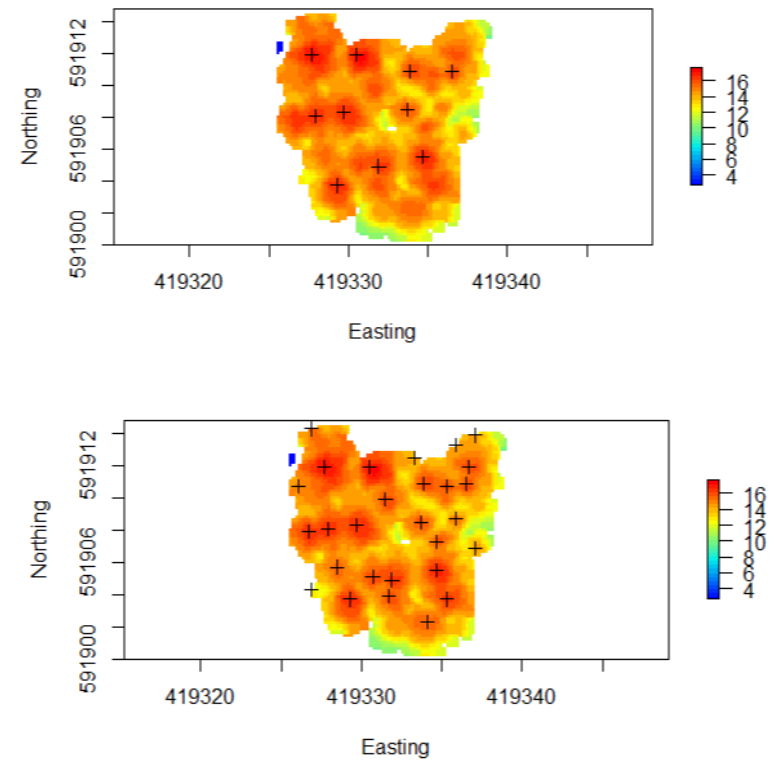

Figure 9. Canopy Height Model of Plot E with detected tree tops. Top: moving kernel size $=3 \mathrm{~m}$, bottom: moving kernel size $=1 \mathrm{~m}$.

\section{CONCLUSIONS}

The study explored a number of segmentation algorithms and their performance across a variety of tree species. It established Continuous Adaptive Mean Shift (Method 5) as the most robust method, achieving high level of accuracy in majority of the plots without the need for extensive calibration. As it was the only clustering method tested, future studies should further explore the utility of a range of clustering algorithms for tree segmentation.

The remaining methods were less consistent in term of performance and might be more suitable in a particular setting certain types of vegetation, with specified parameters. Despite the oversimplification of the $3 \mathrm{D}$ point cloud, as a result of $2 \mathrm{D}$ canopy height model calculation, under certain conditions and with appropriate fine-tuning, CHM segmentation methods can achieve good results. Region growing algorithms, both raster and point cloud based, tend to perform better in coniferous plots, as the tree tops are easier to identify. The majority of plots used in this study were homogeneous and only one plot included a mix of two broadleaf species. Future studies would benefit from including more heterogeneous plots, such as mix of broadleaf and coniferous species.

\section{REFERENCES}

Balsi, M., Esposito, S., Fallavollita, P. \& Nardinocchi, C., 2018. Single-tree detection in high-density LiDAR data from UAVbased survey. Eur. J. Remote Sens. 51, 679-692.

Berra EF, Gaulton R, Barr SL., 2019. Assessing spring phenology of a temperate woodland: A multiscale comparison of ground, unmanned aerial vehicle and Landsat satellite observations. Remote Sensing of Environment. 223, 229-242.

Chen, Q., Baldocchi, D., Gong, P. \& Kelly, M., 2006. Isolating Individual Trees in a Savanna Woodland Using Small Footprint Lidar Data. Photogramm. Eng. Remote Sens. 72, 923-932.

Dalponte, M. \& Coomes, D. A., 2016. Tree-centric mapping of 
forest carbon density from airborne laser scanning and hyperspectral data. Methods Ecol. Evol. 7, 1236-1245.

Eysn, L., Hollaus, M., Lindberg, E., Berger, F., Monnet, J. M., Dalponte, M., Kobal, M., Pellegrini, M., Lingua, E., Mongus, D. \& Pfeifer, N., 2005. A benchmark of lidar-based single tree detection methods using heterogeneous forest data from the Alpine Space. Forests 6, 1721-1747.

Fassnacht, F. E., Latifi, H., Stereńczak, K., Modzelewska, A., Lefsky, M., Waser, L. T., Straub, C. \& Ghosh, A., 2016. Review of studies on tree species classification from remotely sensed data. Remote Sens. Environ. 186, 64-87.

Ferraz, A., Bretar, F., Jacquemoud, S., Gonçalves, G., Pereira, L., Tomé, M. \& Soares, P., 2012. 3-D mapping of a multi-layered Mediterranean forest using ALS data. Remote Sens. Environ. $121,210-223$.

Ferraz, A., Saatchi, S., Mallet, C. \& Meyer, V., 2016. Lidar detection of individual tree size in tropical forests. Remote Sens. Environ. 183, 318-333.

Gupta, S., Weinacker, H. \& Koch, B., 2010. Comparative analysis of clustering-based approaches for 3-D single tree detection using airborne fullwave LIDAR data. Remote Sens. 2, 968-989.

Hamraz, H., Contreras, M. A. \& Zhang, J., Arobust approach for tree segmentation in deciduous forests using small-footpring airborne LiDAR data. Int. J. Appl. Earth Obs. Geoinf. 52, 532541.

Hyyppä, J., Kelle, O., Lehikoinen, M. \& Inkinen, M., 2001. A Segmentation-Based Method to Retrieve Stem Volume Estimates from 3-D Tree Height Models Produced by Laser Scanners. IEEE Trans. Geosci. Remote Sens. 39, 969-975.

Kaartinen, H., Hyyppä, J., Yu, X., Vastaranta, M., Hyyppä, H., Kukko, A., Holopainen, M., Heipke, C., Hirschmugl, M., Morsdorf, F., Næsset, E., Pitkänen, J., Popescu, S., Solberg, S., Wolf, B. M. \& Wu, J. C., 2012. An international comparison of individual tree detection and extraction using airborne laser scanning. Remote Sens. 4, 950-974.

Kandare, K., Ørka, H. O., Chan, J. C. W. \& Dalponte, M., 2016. Effects of forest structure and airborne laser scanning point cloud density on 3D delineation of individual tree crowns. Eur. J. Remote Sens. 49, 337-359.

Li, W., Guo, Q., Jakubowski, M. K. \& Kelly, M., 2012. A New Method for Segmenting Individual Trees from the Lidar Point Cloud. Photogramm. Eng. Remote Sens. 78, 75-84.

Lindberg, E., Eysn, L., Hollaus, M., Holmgren, J. \& Pfeifer, N., 2014. Delineation of tree crowns and tree species classification from full-waveform airborne laser scanning data using 3-d ellipsoidal clustering. IEEE J. Sel. Top. Appl. Earth Obs. Remote Sens. 7, 3174-3181.

Pollock, R. J., 1994. Model-based approach to automatically locating tree crowns in high spatial resolution images. Image and Signal Processing for Remote Sensing. 2315. International Society for Optics and Photonics.

Roussel, J-R., Auty, D., De Boissieu, F., Sanchez Meador, A.,
2019. lidR: Airborne LiDAR Data Manipulation and Visualization for Forestry Applications. R package v 2.0.2.

Silva, C. A., Hudak, A. T., Vierling, L. A., Loudermilk, E. L., O'Brien, J. J., Hiers, J. K., Jack, S. B., Gonzalez-Benecke, C., Lee, H., Falkowski, M. J. \& Khosravipour, A., 2016. Imputation of Individual Longleaf Pine (Pinus palustris Mill.) Tree Attributes from Field and LiDAR Data. Can. J. Remote Sens. 42, 554-573.

Solberg, S., Naesset, E. \& Bollandsas, O. M., 2006. Single tree segmentation using airborne laser scanner data in a structuraly heterogeneous spruce forest. Photogramm. Eng. Remote Sens. $72,1369-1378$

Vauhkonen, J., Ene, L., Gupta, S., Heinzel, J., Holmgren, J., Pitkänen, J., Solberg, S., Wang, Y., Weinacker, H., Hauglin, K. M., Lien, V., Packalén, P., Gobakken, T., Koch, B., Næsset, E., Tokola, T. \& Maltamo, M., 2012 Comparative testing of singletree detection algorithms under different types of forest. Forestry $85,27-40$.

Xiao, W., Elberink, S. O. \& Vosselman, G., 2016. Individual Tree Crown Modeling and Change Detection From Airborne Lidar Data. Ieee J. Sel. Top. Appl. Earth Obs. Remote Sens. 111 . 